\title{
A computational drug design strategy against the Yellow Fever Virus helicase
}

\author{
Eleni Papakonstantinou ${ }^{1 凶}{ }^{\bowtie}$, Katerina Pierouli ${ }^{1}$, Dimitrios Vlachakis ${ }^{1,2,3}$, George N. Goulielmos ${ }^{4}$, Elias Eliopoulos ${ }^{1}$
}

${ }^{1}$ Laboratory of Genetics, Department of Biotechnology, School of Applied Biology and Biotechnology, Agricultural University of Athens, Athens, Greece

${ }^{2}$ Laboratory of Molecular Endocrinology, Center of Clinical, Experimental Surgery and Translational Research, Biomedical Research Foundation of the Academy of Athens, Athens, Greece

${ }^{3}$ School of Informatics, Faculty of Natural \& Mathematical Sciences, King's College London, London, United Kingdom

${ }^{4}$ Section of Molecular Pathology and Human Genetics, Department of Internal Medicine, School of Medicine, University of Crete, Heraklion, Greece

Competing interests: EP none; KP none; DV none; GNC none; EE none

Yellow Fever is an acute viral hemorrhagic disease transmitted through infected Aedes species mosquitoes. It causes fever, bleeding, shock, heatstroke, liver, kidney and myocardial damage and unfortunately, has a high mortality rate. While the Yellow Fever Virus (YFV) affects mainly parts of South America and Africa, in recent years, cases of infection on both animals and humans have been reported in North America, Asia and Europe. A potential YFV infection could have a significant impact on the health of the population, the economy, and the wellbeing of a country. We present a computational strategy for developing novel antiviral inhibitors that target the enzymatic activity of the YFV helicase. We use a holistic bioinformatic approach to enhance our understanding of the YFV helicase enzyme mode and design a series of compounds as candidate drugs against the endemic YFV virus. Phylogenetic studies and structural analyses of the viral helicase and RNA-helicase complex are performed to design a $3 \mathrm{D}$ pharmacophore that will incorporate all physicochemical properties essential for interaction and will be used for high throughput virtual screening and the identification of lead compounds. The in silico pipeline will be evaluated and optimised after in vitro experimental studies aiming to indicate the optimal precursors and their respective moieties. In this way, the in silico pipeline will allow for the discovery of the most potent molecules with an inhibitory effect on the YFV helicase function and the viral replication cycle.

\section{Acknowledgements}

This work is funded by the ESPA Young Researchers Support, «Rational Drug Design of Novel Antiviral Agents against the Helicase Enzyme of the Yellow Fever Virus», MIS 5048546, NSRF 2014 - 2020 